\title{
La transversalización de las matemáticas como base para el cambio conceptual en física. Experiencia en educación inicial ${ }^{1}$
}

\author{
The mainstreaming of mathematics as a basis for conceptual \\ change in physics. Experience in early childhood education
}

\section{A integração da matemática como base para a mudança conceitual na física. Experiência na educação infantil}

Recibido: mayo de 2013

Aceptado: agosto de 2013
Jorge Eliécer Villarreal Fernández ${ }^{2}$ María Elena Tapia ${ }^{3}$

\section{Resumen}

El desarrollo de las habilidades de pensamiento en los niños, que permitan un acercamiento más claro a la comprensión de los fenómenos físicos que se manifiestan en su entorno, es una de los objetivos que la educación inicial se plantea. Para el cumplimiento de este fin es necesario que otras áreas del conocimiento se transversalicen en el desarrollo de las actividades de enseñanza de los procesos físicos. Este trabajo muestra cómo se relacionaron diversos conceptos matemáticos durante un proyecto de aula que buscaba el acercamiento de los estudiantes al conocimiento actuando como científicos naturales, y cómo estos conceptos actuaron como herramientas esenciales en el proceso de cambio de conceptos en los niños al ser artífices del conflicto cognitivo.

Palabras clave: Cambio conceptual; educación inicial; transversalización; conflicto cognitivo; matemáticas; matemáticas y otras disciplinas.

\begin{abstract}
Developing thinking skills in children, which allow a more clear understanding of the physical phenomena that occur in your environment, is one of the objectives that early education arises. To fulfill this purpose it is necessary that other areas of knowledge in developing transversalicen teaching activities of the physical processes. This paper shows how various mathematical concepts related for a classroom project that sought the views of the students to act as natural scientific knowledge, and how these concepts act as essential tools in the process of changing concepts in children to be creators of cognitive conflict.
\end{abstract}

Key Words: Conceptual change; initial education mainstreaming; cognitive conflict, mathematics, mathematics and other disciplines.

1 Artículo de Investigación.

2 Politécnico Colombiano Jaime Isaza Cadavid, Colombia. Contacto: jorgevf2005@gmail.com,

3 Jardín de Infantes N 18 "Islas Malvinas Argentinas" Caleta Olivia- Santa Cruz, Argentina, Contacto: marelen26200@hotmail.com 


\section{Resumo}

Desenvolver habilidades de pensamento em crianças, o que permite uma compreensão mais clara dos fenômenos físicos que ocorrem em seu ambiente, é um dos objectivos que a educação surge. Para cumprir este objetivo, é necessário que outras áreas do conhecimento para o desenvolvimento de atividades de ensino transversalicen dos processos físicos. Este artigo mostra como vários conceitos matemáticos relacionados para um projeto de sala de aula que procurou saber a opinião dos alunos para atuar como conhecimento científico natural, e como esses conceitos funcionam como ferramentas essenciais no processo de mudança de conceitos em crianças a serem criadores de conflito cognitivo.

Palavras chave: Mudança conceitual; integração formação inicial; conflito cognitivo, matemática, matemática e outras disciplinas.

\section{Contextualización}

Esta experiencia de aula surge a partir de una salida recreativa en donde los niños recorrieron distintos lugares de la ciudad de Caleta Olivia (Centro - Costanera: plazas y playones) con la finalidad de recrearse al aire libre y compartir un momento ameno. Al llegar a la costanera los niños divisaron a lo lejos un barco de gran tamaño, el cual centralizó su atención, la observación espontánea del mismo llevó al surgimiento de diversos interrogantes entre ellos: ¿Quiénes viven allí? ¿Por qué no se hunde? ¿Por qué flota? ¿Tiene un capitán? ¿Qué hacen las personas allí? ¿Qué tiene adentro?, etc. Si bien esa no era la intención de la salida, el interés manifestado por los niños quedó pendiente para recuperarlo y retomarlo en el momento apropiado para su abordaje.

Es por ello que pareció relevante recuperar el interrogante: ¿Por qué no se hunden los barcos? ¿Por qué flotan? Dado que el mismo no sólo permite transformarlo en una situación problemática-objeto de estudio sino que a su vez permitirá a los niños la búsqueda de respuestas enfocadas desde las Ciencias Naturales mediante la implementación del método experimental acercándolo a la Física. Los niños podrán experimentar con diversos objetos, anticipar sus hipótesis iniciales, refutarlas, compararlas y reelaborar con la finalidad de buscar una aproximación de respuesta a la duda generada. Este acercamiento a partir de actividades de experimentación permitió que los niños utilizaran herramientas matemáticas necesarias para que cada una de las experiencias pudiera materializarse y determinarse en ellas las conjeturas iniciales que se habían planteado.

\section{Referentes teórico-prácticos}

Diversas investigaciones a nivel mundial muestran que la enseñanza de las matemáticas en edades tempranas se convierte en un importante medio para predecir niveles de logro en el área en grados superiores, es decir los aprendizajes matemáticos en la infancia son base de la estructuración del pensamiento matemático en años posteriores (Cerda, Pérez, Ortega, Lleujo \& Sanhueza, 2011). Estos desarrollos se presentan a medida que la formación va realizándose de una manera más integral, es decir a partir del desarrollo de las dimensiones del ser humano. Una de estas dimensiones es la cognitiva la cual tiene que ver con el problema del conocer de los sujetos. El conocimiento es construido por los que aprenden y no por los que enseñan, dado que no se aprende pasivamente sino a través de la actividad. El interactuar con el alumno debe ayudarlo a configurar sus estructuras y procesos mentales (Villarreal, Mestre \& Llanez, 2011). El desarrollo de la dimensión cognitiva ha tenido como base las áreas del conocimiento y en particular las matemáticas ya que en ella es donde se posibilita el desarrollo de los procesos de pensamiento tales como analizar, describir, comparar, deducir, 
inducir, entre otras; y por ende ayuda a desarrollar las capacidades mentales del individuo, por lo que la persona podrá conocer y descubrir el conocimiento. Desde esta perspectiva, ha sido mucho el aporte de las matemáticas al desarrollo social, cultural y económico de la humanidad que justifica que sean parte obligada de la formación integral del individuo.

Villarreal et al. (2011) plantean que la utilización de la lógica como principio de los conceptos verdaderos permite formar un hombre organizado, responsable, crítico, analítico, justo, equitativo y tolerante, con capacidad para desarrollar políticas que permitan plantear y solucionar problemas personales, comunes, sociales contribuyendo al beneficio personal, regional y nacional, es decir permite el aprender a vivir con los demás, juntos y a ser.

Por otra parte la aplicación de nuevas herramientas y técnicas frente a la construcción del conocimiento y el desarrollo de la ciencia misma como son los computadores y las calculadoras en la utilización de programas de cálculo, geometría plana, espacial $\mathrm{y}$ vectorial, plantean un nuevo reto entre la generación actual y la máquina, que llevan a un aprender a hacer (Villarreal et al., 2011).

Teniendo en cuenta lo anterior, es importante la transversalización, es decir el que los temas matemáticos atraviesen los contenidos curriculares de la educación inicial, de tal forma que sirvan como base para la contextualización y se conviertan en instrumento para el logro de otras finalidades (Fernández, 2000). En el caso de este trabajo el proceso de transversalizar contenidos matemáticos en la aproximación a los conceptos físicos busca que se conviertan en instrumento para generar conflictos cognitivos en el estudiante y, de esta manera, dar pie al docente para que indague sobre nuevas alternativas que propicien el acercamiento a la comprensión de los fenómenos y al desarrollo de habilidades de pensamiento en el niño.

\section{Experiencia en el aula}

La experiencia hace parte de un proyecto de aula que tenía como objetivo el acercamiento al concepto de flotabilidad por parte de niños de preescolar pertenecientes al Jardín de Infantes No 18, "Islas Malvinas Argentinas". El proyecto tuvo como punto de partida la observación del entorno de donde surgen las inquietudes que se sintetizan en la pregunta ¿Por qué flotan los barcos?, y las diferentes conjeturas iniciales que plantearon los estudiantes en el intento por resolver el interrogante.

A partir de estas conjeturas se diseñaron actividades donde se pudieran poner a prueba las ideas que se tienen sobre el fenómeno. La primera actividad consistió en organizar una serie de elementos que iban a ser metidos en agua para comprobar la flotación de cada uno de ellos. Antes de iniciar la actividad experimental los estudiantes realizaron tablas de datos donde una columna tenía las imágenes de los cuerpos a experimentar, otra columna donde se escribía lo que pensaban sucedería con el objeto, otra donde se escribiría el porqué de esta situación y una cuarta columna donde se registró el resultado, es decir lo que de verdad ocurrió. Antes las diferencias en algunas de las predicciones sobresale una explicación, que es por el peso de los objetos que se da la flotabilidad, es decir lo que pesa menos flota, lo que pesa más se hunde.

A partir de esto se llevó una balanza electrónica al aula, además de nuevos elementos de experimentación entre los que se encontraban botellas de plástico con arena en su interior. Cada uno de estos elementos fue pesado y registrado los datos en otra tabla con las mismas columnas que la anterior, sumándole la del peso de los objetos utilizados. No se cumplían los planteamientos, los cuerpos que pesaban más no se hundían (botellas) y los menos pesados si se hundían (agujas, bolas de cristal, etc.), ante estos resultados los estudiantes plantearon que la balanza estaba dañada, que el problema seguía siendo el peso. Se vuelven a realizar las mediciones utilizando otra balanza y la experimentación en el agua. Nuevamente un conflicto entre lo que se pensaba iba a ocurrir y lo ocurrido. Ante esto el consenso de los niños fue que la forma era la característica que permitía que los objetos flotaran.

Para poner a prueba esta nueva conjetura, se trae plastilina que se le ha dado forma de esfera y que al ser metida en el agua también se hunde. Los niños dibujan las formas iniciales y comienzan a 
manipularlas para introducirlas al agua y comprobar la conjetura planteada. Antes de meter al agua la plastilina dibujan la nueva forma y registraron en una tabla lo que ocurrió. Después de varios intentos logran hacer que la plastilina flote, con esto comprueban lo que habían anticipado. A partir de este logro se plantea que existe algo dentro del agua que impulsa hacía afuera a los objetos que se introducen en ella. A partir de esta última afirmación se realizan otras actividades de comprobación de lo dicho y se realiza una formalización de lo que sería la fuerza de empuje a partir de imágenes que mostraban el comportamiento de ese "algo" que los niños habían descubierto. Para culminar el proceso se contó la historia de Arquímedes y se realizó una representación dramática de la anécdota de la corona y el grito de ¡Eureka! Y su significación. La dramatización fue realizada por los estudiantes.

\section{Logros y dificultades evidenciadas}

El principal logro a nivel del planteamiento físico fue el descubrimiento de la fuerza de empuje por parte de los estudiantes, además de esto la identificación de algunas de las características del fenómeno de flotabilidad. Se encaminó al estudiante hacía la búsqueda del conocimiento como un científico natural, desarrollándose a partir de esto habilidades de pensamiento como la percepción, la observación, la identificación, la codificación, la descripción, la comparación, el análisis, la relación, clasificación, todo esto en el proceso de resolución de las preguntas iniciales a través de actividades experimentales (MECyT, 2004a).

A nivel matemático los estudiantes lograron el reconocimiento del número en contextos de conteo y medición. A partir de este reconocimiento pudieron describir situaciones diferentes y realizar comparaciones. Diferenciaron características de objetos tridimensionales y dibujaron y describieron diversos cuerpos en distintas posiciones y tamaños. En los objetos se reconocieron atributos y propiedades que se podían medir, la longitud, determinando cuando un cuerpo era más grande que otro y el peso, llegando a reconocer cuando un cuerpo pesaba más que otro. Todos los datos fueron clasificados y organizados en tablas y se realizó la interpretación de lo que allí se consignó (MECyT, 2004b). A partir del conjunto de datos en la tabla se describieron los eventos que ocurrían, las diferencias con lo esperado y se realizaron predicciones sobre lo que podía suceder en nuevas actividades experimentales.

A partir de los resultados que se iban obteniendo se presentaban conflictos cognitivos. Las ideas iniciales no funcionaban ya que los datos arrojados por las actividades experimentales y consignadas en las tablas, o aquellas transformaciones de las formas de los objetos, no eran semejantes a las esperadas, esto llevaba a la necesidad de plantear nuevas conjeturas y volver a utilizar la herramienta matemática para describir varios de los eventos y sobre esta base comprobar o no las anticipaciones de los estudiantes. La transversalización de la matemática en esta situación presenta un nuevo logro, el saber que se puede aplicar en el contexto físico, lo que permite un adecuado acercamiento al conocimiento matemático desde lo conceptual, lo procedimental y lo actitudinal y se convierte en base para el cambio en las concepciones iniciales de los estudiantes sobre el fenómeno estudiado.

El proyecto fue segundo lugar en la Feria de la Ciencia del municipio y mención de honor a nivel provincial. En estos eventos fueron los mismos estudiantes los que explicaban lo que había sucedido al público participante, mostrándose un desarrollo a nivel comunicativo y de explicación de los procesos.

Las dificultades presentadas tuvieron que ver con la falta de competencias en lectura y escritura de algunos estudiantes, que se resuelven a partir del manejo de imágenes, lo que se vio reflejado en el diseño de las tablas de datos. La complejidad del concepto de flotabilidad es un obstáculo para su comprensión a esta edad, por lo que se definió centrar en el desarrollo de las habilidades de pensamiento que permitirían tener una base cognitiva adecuada para que, en el futuro, el concepto pueda ser comprendido de manera completa.

\section{Reflexión final}

La transversalización de las matemáticas en actividades de otras áreas (cómo la física en este caso) es una 
herramienta esencial para la comprensión de algunas de las características de fenómenos que le son desconocidos al estudiante. Además se logra contextualizar los conceptos matemáticos consiguiendo un mejoramiento del proceso de aprendizaje de los mismos, ya que se convierten en elementos cercanos para el estudiante. El cambio en los conceptos se realiza a medida que la evidencia que la matemática genera pone en conflicto las concepciones iniciales de los estudiantes, es por esto que la matemática cumple aquí un doble papel, el de conocimiento como tal, propio, y como herramienta para la comprensión de conocimientos de otras ciencias, como la física. A partir de los resultados, también se puede ver cómo el niño tiene capacidades que en muchas ocasiones no se tienen en cuenta, desaprovechando edades que son claves para que los procesos de desarrollo a nivel neuronal se puedan dar, la matemática en este caso cumple también un papel de objetivo de aprendizaje y de posibilidad para generar nuevos. Desde los años iniciales de educación se pueden realizar propuestas de enseñanza de las diversas ciencias a partir de actividades que integren los diferentes campos del conocimiento, lo que permite que el niño, quien tiene una mente global en ese momento, tenga facilidad para acceder a las nociones a aprender. Además de esto es importante el inicio desde situaciones concretas, ya que el pensamiento del niño es edades tempranas aún mantiene esta condición de concreto.

\section{Referencias}

Cerda, G., Pérez, C., Ortega, R., Lleujo, M. \& Sanhueza, L. (2011). Fortalecimiento de competencias matemáticas tempranas en escolares, un estudio chileno. Psychology, Society, \& Education, 3(1), 23-29.

Fernández, J. (2000). Una escuela para todos desde la transversalidad: los Contenidos Transversales. En A. Miñambres y G. Jové. (Coords), La atención a las necesidades educativas especiales: de la Educación Infantil a la Universidad. Lleida: Universidad de Lleida, 493-498.

Ministerio de Educación, Ciencia y Tecnología. (2004a). Núcleos de Aprendizaje Prioritarios. Nivel Inicial. MECyT: Buenos Aires.

Ministerio de Educación, Ciencia y Tecnología. (2004b). Núcleos de Aprendizaje Prioritarios. Nivel Inicial. MECyT: Buenos Aires.

Villarreal, J., Mestre, U., \& Llanez, L. (2011). La atención a las diferencias individuales, en aulas inclusivas, como vía para el aprendizaje desarrollador de las matemáticas en la educación básica y media en Colombia.Didasc@lia: Didáctica y Educación, (4), 59-74. 